\title{
An advanced laboratory management software
}

\author{
Dr. M.H.B. Ariyaratne \\ Postgraduate Institute of Medicine, University of Colombo, Sri Lanka \\ E-mail address: buddhika.ari@gmail.com
}

eHealth Sri Lanka 2010,1(suppl.1):S29

DOI: http://dx.doi.org/10.4038/sljbmi.v1i0.3587

Only the Abstract is available

\begin{abstract}
A laboratory management software has become an essential equipment even in small scale laboratories in Sri Lanka. Its usage exceeds those of EHRs and Pharmacy Software. This paper is a description of a widely used Laboratory Management Software in Southern Province of Sri Lanka. Software can handle a virtually unlimited number of investigations. Several departments are managed simultaneously. There is complete freedom to the end user to do any change in the report format. The prices, material usage, costs, fees for staff, the department, the sample and quantity needed are all recorded alone with each investigation. The issuing of the bill at the reception can be configured to contain the instruction regarding the technical details like the tube to be used to collect the sample. The details are updated in the relevant department real-time. After the test is performed, the entering of the results is very efficient as a default set of results can be configured to appear when a new report is going to be generated, and only the changes are needed to be updated. Even changing the values of a certain field of an investigation is very easy as the probable values are listed and it is just a matter of selecting on of them. The default format and the list of possible values can be changed himself by the MLT without the help of the developer. Even the new additions in to a certain field can automatically gather new values to be listed in future usage if selected. The retrieval of patient details, referring doctor's details, the results of all the investigation, the financial transactions and material usage is very easy and can be analysed when required.
\end{abstract}

Keywords - laboratory management software, freedom to the end user 